\title{
EFFECTS OF ACOUSTICALLY LINED CYLINDRICAL DUCTS ON INSTABILITY WAVES IN CONFINED SUPERSONIC JETS
}

\author{
Milo D. Dahl* \\ NASA Lewis Research Center \\ Cleveland, $\mathrm{OH} 44135$
}

\begin{abstract}
The pressure disturbances generated by an instability wave in the shear layer of a supersonic jet are studied for an axisymmetric jet inside a lined cylindrical duct. For the supersonic jet, locally linear stability analysis with duct wall boundary conditions is used to calculate the eigenvalues and the eigenfunctions. These values are used to determine the growth rates and phase velocities of the instability waves and the radial pressure disturbance patterns. The study is confined to the dominant Kelvin-Helmholtr instability mode and to the region just downstream of the nozzle exit where the shear layer is growing but is still small in size compared to the radius of the duct. Numerical results are used to study the effects of changes in the outer flow, growth in the shear layer thickness, wall distance, wall impedance. and frequency. Results indicate that the effects of the duct wall on shear layer growth rates diminish as the outer flow increases. Also, wall reflections (ause variations in growth rates depending on wall height and Strouhal number. These variations are due to the phase relationship between the outgoing and the reflected incoming pressure disturbances at the shear layer. The growth rate variations can be reduced and the maximum growth rate minimized by keeping the imaginary part of the impedance negative.
\end{abstract}

\section{INTRODUCTION}

A confining wall affects the supersonic jet mixing layers that are associated with combustion proresses.' engine tests conducted inside an enclosed facility,${ }^{2}$ and jet noise reduction efforts. ${ }^{3}$ In the circular configuration considered for this paper, the supersonic jet issues from a nozzle that has its axis coincident with the centerline of a surrounding cylindrical duct. Between the jet and the confining wall of the duct, a lower speed outer flow exists. The shear layer between the two flows is initially thin and unstable. This instability grows like a wave as the fow moves downstream. As a result, this instability wave generates perturbations that propagate away from the shear layer. Those perturbations that propagate toward the wall can reflect off it and return toward the shear laver to interact and modify the growing instability in the shear layer. Thus the presence of the wall, both if the wall is hard and if it has an acoustic lining. affects the characteristics of the instability waves that govern both the mixing in the high speed shear layer and the noise generation.

Stability analysis was used by Tam and $\mathrm{Hu}^{4}$ to study the supersonic mixing layer inside a rectangular channel with hard walls. In addition to the Kelvin-Helmholtz ( $\mathrm{KH}$ ) instabilities, they found from using a vortex sheet model that the coupling of the unsterady motion with acoustic modes reflected from the walls resulted in a new family of supersonic instabilities with supersonic convertive Mach number. The analysis also included the effects of shear layer thickness on the spatial growth rates of twoand three-dimensional supersonic instability waves. Zhuang et al." showed for a constant shear laver thickness that the walls most affected the shear laver instability when the convective Mach number was greater than one. As the distance between the walls decreased, the maximum amplification rate reached a maximum value and then decreased. The stability characteristics for subsonic convective Mach number waves were unaffected by wall height. Others have found similar results for shear layers in a rectangular channel . $1.6 \mathrm{i}$

The stability analysis has been performed for a cylindrical supersonic vortex sheet inside' a cylindrical duct with hard walls. ${ }^{2,7-9}$ Viswanathan ${ }^{7}$ showed how the $\mathrm{KH}$ instabilities for a $M_{j}=4.0$ jet with no outer flow were affected by the wall height. The effect appeared as oscillations in the growth rates due to interaction between the internal Mach wave system of the jet and the reflected waves from the wall as the wall moved closer to the vortex sheet. Furthermore, the $\mathrm{KH}$ mode had growth rates typically higher than the supersonic instabilities. Chang and Kuo found similar results for a $M_{j}=4.5$ jet with two different outer flows. $M_{0}=4.06$ and $M_{0}=1.56$. 
It was also shown that the axisymmetric $\mathrm{KH}$ mode growth rates decreased as the outer flow velocity increased relative to the inner flow velocity. When shoar laver thickness was included in the analysis. the growth rates for the instabilities were diminished and the presence of the hard wall had a greater affect on KH mode growth rates than on supersonic: mode growth rates. 7

$\mathrm{Hu}^{3}$ recently studied the effects of two parallet walls with sound absorbing lining on a twodimensional jet confined inside a rectangular duct. The analysis was performed for two-dimensional instability waves with mean flow conditions $M_{j}=2.0$, $M_{o}=0.2$, and $T_{j} / T_{n}=4.0$. Using a vortex sheet model, the lined walls were shown to be most effective when the phase velocity was supersonic relative to the outer flow and when the pressure eigenfunction showed large perturbations near the wall. In general, growth rates were reduced when the wall impedance was finite. A continuous mean flow model based on a hyperbolic tangent function was used to include the effects of shear layer thickness. For the instability wave with the largest growth rates, the increasing shear layer thickness resulted in lower growth rates. Also, the presence of a lined wall was more effective in reducing growth rates than a hard wall.

In this paper, we extend the study of the effects of a confining wall with sound absorbing lining on shear layer instability waves to the cylindrical duct case. Many variables are available to study including the inner jet stream velocity and temperature, the outer stream velocity and temperature, the axial location, the jet shear layer width, the duct wall height, the wall impedance both real and imaginary parts, and the frequency. By necessity, we must limit our presentation of results. The jet Mach number is fixed at 2.0 with a temperature ratio $T_{j} / T_{o}=2.0$. We are primarily interested in the application of acoustic wall treatment in an ejector designed to reduce supersonic jet noise. Inside the ejector, a shear laver between the two flow streams exists only for a short distance downstream of the nozzle exit before the two flows more fully mix together. Consequently, the results presented herein will be confined to small shear layer widths near the nozzle. Thus, this study concentrates on the effects of wall treatment on the initial growth characteristics of the shear layer. We will only consider the Kelvin-Helmholtz type mode in this study since it is the dominant instability for both the free and confined jets at the above flow conditions. The next section describes the formulation of the instability wave model using a finite impedance boundary condition. The model is used to determine the growth rates and phase velocities of the instability waves that govern the pressure disturbance pattern generated by the jet shear layer. This is followed by a presentation and a discussion of the numerical results.

\section{INSTABILITY WAVE FORMULATION}

A cross-section of the model configuration for a. supersonic axisymmetric jet inside a cylindrical duct is shown in Figure 1. The high speed flow emerging from the nozzle with velocity $U_{j}$ and static: temperature $T_{j}$ has an initially thin shear layer that is inherently unstable even in the absence of viscosity. An instability wave begins to grow rapidly and contimues to grow at reduced rates as the shear laver spreads. During this process, pressure disturbances created by the instability propagate away from the shear layer toward the wall, reflect off the wall, and return to interact with and modify the instability wave in the shear layer. This process is assumed to be governed by the linearized, inviscid, compressible equations of motion.

\section{Disturbance Equations}

For a supersonic jet, the shear layer grows slowly in the axial direction. This allows a locally paralle] How approximation to be used to obtain the solution for the disturbance quantities. The fluctuating disturbances are represented as waves traveling through a nomuniform medium. As an example, the pressure disturbances are given by

$$
\begin{aligned}
p^{\prime}(r, \theta, x, t) & =p(r, x) \\
& \times \exp \left[i\left(\int_{0}^{x} \alpha(\chi) d \chi+n \theta-\omega t\right)\right](1)
\end{aligned}
$$

where $p(r, x)$ represents the radial distribution of the pressure disturbance at each axial location, $\alpha(x)$ is the local complex wave number $\left(\alpha=\alpha_{r}+i \alpha_{i}\right.$ and $-\alpha_{i}$ is the local growth rate), $\theta$ is the azimuthal angle, $n$ is the mode number, and $\exp (-i \omega t)$ is the harmonic time dependence. The other disturbance quantities have the same form. The linearized equations governing the disturbances can be combined to obtain a single equation called the compressible

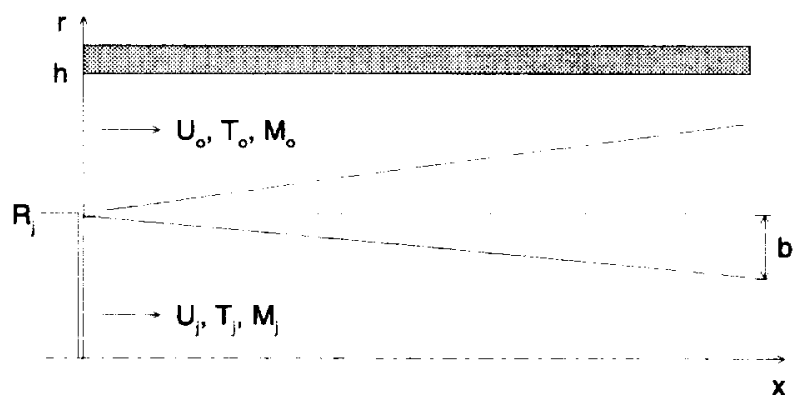

Figure 1: Schematic diagram in the $x-r$ plane of an axisymmetric jet flowing into a cylindrical duct with coflowing outer stream. 
Rayleigh equation,

$$
\begin{aligned}
\frac{\partial^{2} p}{\partial r^{2}} & +\left[\frac{1}{r}+\frac{2 \alpha}{\omega-\alpha \bar{u}} \frac{\partial \bar{u}}{\partial r}-\frac{1}{\bar{\rho}} \frac{\partial \bar{p}}{\partial r}\right] \frac{\partial p}{\partial r} \\
& +\left[\bar{\rho} M I_{j}^{2}(\omega-\alpha \bar{u})^{2}-\frac{n^{2}}{r^{2}}-\alpha^{2}\right] p=0 .
\end{aligned}
$$

This equation has been nondimensionalized as follows: spatial coordinates by $R_{j}$, time and radial frequency by $U_{j} / R_{j}$, velocity by $U_{j}$, density by $\rho_{j}$, and pressure by $\rho_{j} U_{j}^{2}$ where the subscript $j$ indicates jet exit conditions. To solve this equation. the radial variation of the mean flow velocity $\bar{u}$ and density $\bar{p}$ must be known quantities provided from either an analytic expression or a numerical calculation.

The general solution to equation (2) at any axial location is written

$$
p(r)=A \zeta_{1}^{p}(r)+B \zeta_{2}^{p}(r)
$$

using unknown functions with radial variations. Outside the jet, the mean flow becomes uniform and equation (2) takes the form of Bessel's equation. The solution can be readily written as

$$
p_{o}=A_{o} H_{n}^{(1)}(i \lambda(\alpha) r)+B_{o} H_{n}^{(2)}(i \lambda(\alpha) r)
$$

where

$$
\lambda(\alpha)=\left[\alpha^{2}-\bar{\rho}_{0} M_{j}^{\prime 2}\left(\omega-\alpha \bar{\mu}_{0}\right)^{2}\right]^{1 / 2} .
$$

$H_{n}^{(1)}$ is a Hankel function of the first kind. It represents a wave outgoing from the shear layer toward the wall. $H_{n}^{(2)}$ is a Hankel function of the second kind and represents the wave reflected from the wall. As $r \rightarrow 0$, equation (3) must be finite.

At the wall, the continuity of particle displacement is used as the boundary condition. ${ }^{3}$ The kinematic equations are combined in terms of pressure to get

$$
\left(\omega-\alpha \bar{u}_{o}\right)^{2} p_{o}+\frac{i \omega}{\bar{\rho}_{o}} \frac{Z^{*}}{\rho_{j} U_{j}} \frac{\partial p_{o}}{\partial r}=0
$$

where $Z^{*}$ is the dimensional acoustic impedance. The acoustic impedance is usually referenced to a typical density and speed of sound. We use ambient conditions for density $\rho_{a}$ and speed of sound $c_{n}$ to get

$$
\frac{Z^{*}}{\rho_{j} U_{j}}=Z \sqrt{\bar{\rho}_{a}} \frac{1}{M_{j}}
$$

where $Z=Z^{*} / \rho_{a} c_{n}$ is the specific acoustic impedance of the wall treatment. This formulation for the wall treatment condition is based on assuming a locally reacting boundary. Substituting equation (4) into equation (6) and evaluating the resulting equation at the radial location $h$ of the wall for the unknown $B_{o}$, we get the equation

$$
p_{o}=A_{o}\left[H_{n}^{(1)}(i \lambda r)-C(h) H_{n}^{(2)}(i \lambda r)\right]
$$

where

$$
C(h)=\frac{\left(\omega-\alpha \bar{u}_{o}\right)^{2} H_{n}^{(1)}(i \lambda h)-\frac{\omega \lambda Z \sqrt{\bar{p}_{a}}}{\bar{p}_{0} M_{j}} H_{n}^{(1) \prime}(i \lambda h)}{\left(\omega-\alpha \bar{u}_{o}\right)^{2} H_{n}^{(2)}(i \lambda h)-\frac{\omega \lambda Z \sqrt{\bar{p}_{a}}}{\bar{p}_{0} M_{j}} H_{n}^{(2) \prime}(i \lambda h)}
$$

is obtained after substituting $B_{\text {o }}$ back into ecguation (4). As $h \rightarrow \infty$. equation (8) reduces to the free jet "ase 10 and as $Z \rightarrow \infty$, the wall becomes acoustically hard and equation (9) reduces to the appropriate form $H_{n}^{(1) \prime}(i \lambda h) / H_{n}^{(2) \prime}(i \lambda h)$ as found in Ref. [9].

Equation (2) and its boundary conditions create an eigenvalue problem with eigenvalue $a$. The centerline boundary condition at $r=0$ depends on whether the pressure eigenfunction is either axisymmetric, $n=0$, or non-axisymmetric, $n \neq 0$. In equattion form, this is writton as

$$
\begin{aligned}
\frac{\partial p}{\partial r} & =0, n=0 \\
p & =0 . n \neq 0 .
\end{aligned}
$$

A finite difference approximation is used to discretize equation (2). To incorporate the outer boundary condition, the constant $A_{0}$ must be eliminated from equation (8). This is done by using a ratio of the disturbance pressures at the two outer grid points,

$$
\frac{p_{N+1}}{p_{N}}=\frac{H_{n}^{(1)}\left(i \lambda r_{N+1}\right)-C(h) H_{n}^{(2)}\left(i \lambda r_{N+1}\right)}{H_{n}^{(1)}\left(i \lambda r_{N}\right)-C(h) H_{n}^{(2)}\left(i \lambda r_{N}\right)}
$$

where $N$ is the number of grid spacings. The local eigenvalue is found from the resulting diagonal matrix using a Newton-Raphson iteration for refinement. The local eigenfunction. $p(r, x)$. is then determined within a constant using the inverse power method. During this latter calculation, the eigenfunction is normalized at the point where the axial mean flow velocity gradient is a maximum in the radial direction.

\section{Mean Flow}

In order to complete the stability analysis, the mean How properties are needed. Often equations consisting of simple analytic functions are used to describe the mean flow. However, in order to easily account for the effects of velocity and temperature differences across the supersonic jet shear layer on shear layer spreading, a numerically generated mean flow is used. The jet is assumed to be perfectly expanded and for the region of interest near the nozzle, the static pressure is assumed to be constant. The set of compressible, Reynolds averagerl. boundary layer equations with a modified turbulence model is used as the basis for the numerical mean flow analysis. The details of the equation development. the numerical solution, and comparisons of calculated results to measured mean flow data are given in Ref. [11]. 


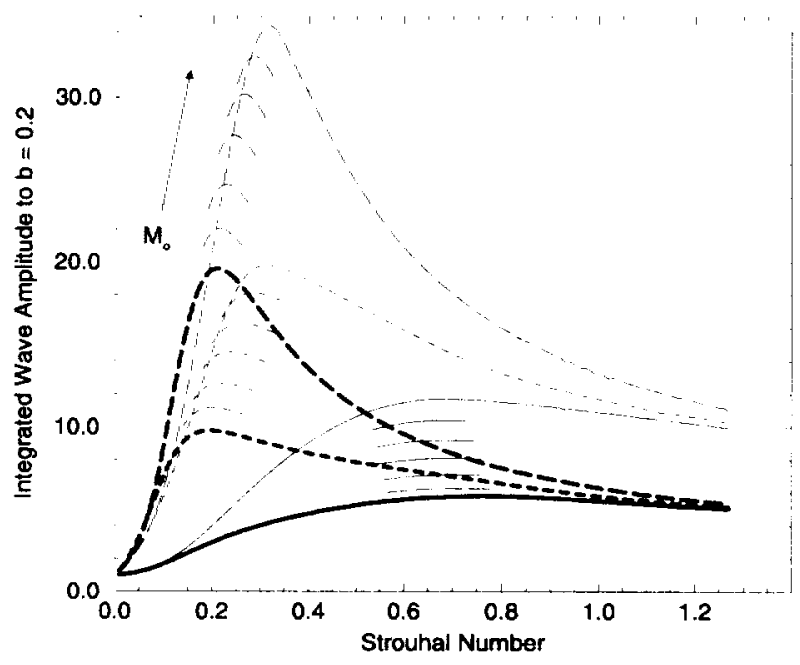

Figure 2: The integrated instability wave amplitudes to the free jet shear layer half-width $b=0.2$. Modes: $(\longrightarrow) n=0 ;(---)) n=1 ;(--\square) n=2$. Outer flow: thick lines, $M_{o}=0.03$; thin lines, $M_{o}=$ 0.6 ; peaks shown for $M_{o}=0.1$ to 0.5 .

\section{NUMERICAL RESULTS}

The calculations were performed for a jet with exit Mach number $M_{j}=2.0$. The jet was hot relative to the outer stream with $T_{j} / T_{o}=2.0$. The outer stream had the same static temperature as the ambient conditions for the source of the outer stream. The velocity ratio between the outer stream and the inner jet stream was varied from 0.01 to 0.21 resulting in outer stream Mach numbers $M_{o}$ of 0.03 and 0.1 to 0.6 in increments of 0.1 for a total of seven operating conditions.

In addition to the flow conditions, the frequency $\omega$ in equation (2) must be given. A study of the instability wave amplitudes for a free jet shear layer calculated from the nozzle to the downstream location where the shear layer half-width $b$ is 0.2 shows peak amplitudes for the $n=1$ and $n=2$ helical modes in the Strouhal number range $\left(S t=\omega / \pi=f D_{j} / U_{j}\right.$ where $D_{j}=2 R_{j}$ ) between 0.2 and 0.3 , see Figure 2 . As the outer flow Mach number increases, the peak wave amplitudes increase and shift slightly to higher Strouhal numbers. Thus, the calculated results will be shown for Strouhal numbers in this peak region. In addition, the results presented in this paper will be for the $n=1$ and $n=2$ instability wave modes since they are clearly more dominant than the $n=0$ axisymmetric instability wave mode.

\section{Hard Wall}

The instability wave growth rates near the nozzle exit are first examined for the hard wall case as a function of the Strouhal number $S t$, the duct wall height $h$, and the outer stream Mach number $M_{0}$. Figure 3 shows growth rate contours at several
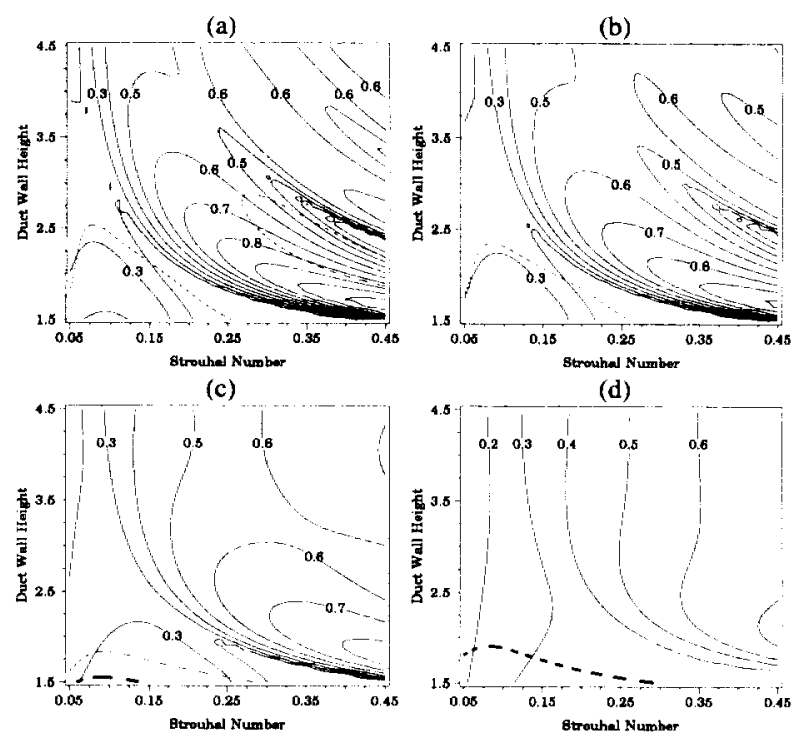

Figure 3: Local growth rate contours as a function of $h$ and $S t$ for the $n=1$ mode of an $M_{j}=2.0$ jet in a hard wall cylindrical duct, $b=0.05$. Outer flow: $M_{o}=$ (a) 0.03 ; (b) 0.1 ; (c) 0.3 ; (d) 0.6 . Phase velocities supersonic relative to outer flow and subsonic relative to inner flow except inside thick dashed line (subsonic re. outer flow) and inside thin dashed line (supersonic re. inner flow).

outer stream Mach numbers for the $n=1$ mode as a function of wall height and Strouhal number at the axial location where the shear layer half-width $b$ is 0.05 . For $M_{o}=0.03$, the growth rates oscillate as both the wall height and Strouhal number change. becoming large near the lower wall height and the largest Strouhal number. This behavior is similar to those growth rate characteristics calculated for a cylindrical vortex sheet. ${ }^{7}$ These growth rate oscillations are due to the location of the wall relative to the standing wave like pattern resulting from reflecting pressure disturbances interfering with the pressure disturbances generated by the shear layer instability. As an example, Figure 4(a) shows the changes in the radial distributions of the wave modes for the local maximum and minimum growth rates as the wall height changes for $S t=0.45$ in Figure 3(a). To indicate the relative amplitude of these modes, the radial distributions are multiplied by their respective local growth rates and then normalized by the growth rate at $h=1.65$. Local nodes and antinodes occur that are due to the interference between outgoing and incoming reflected disturbances. The total pressure disturbance amplitude is always a local maximum at a hard wall. As the reflected disturbance interacts with the shear layer, the growth rate can be enhanced or reduced. Figure $4(\mathrm{~b})$ shows the outgoing and the incoming pressure disturbance patterns separately (note that the pressure distur- 
bance reflects in phase at the wall). The major difference between the instability wave having a local maximum growth rate or a local minimum growth rate is the phase relationship between the outgoing and incoming disturbances. For a local maximum growth rate, the phase differences between the incoming and the outgoing disturbances just outside the shear layer are $-148.5^{\circ},-152.6^{\circ}$, and $-152.6^{\circ}$ when the wall heights are $1.65,2.70$, and 3.75 , re-

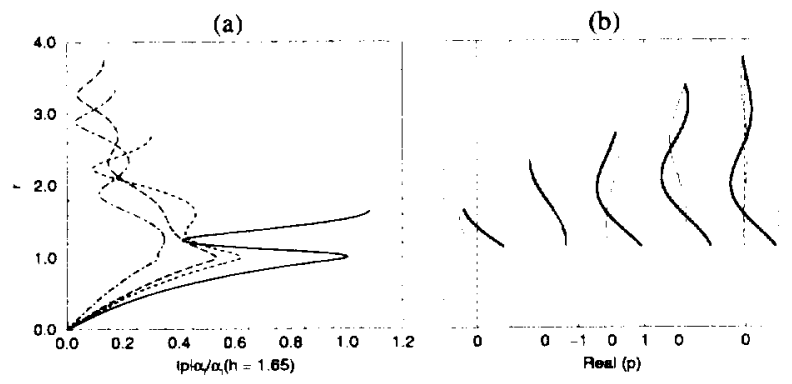

Figure 4: Radial distribution of wave modes for conditions in Figure $3(\mathrm{a}), S t=0.45$. (a) Pressure magnitudes for different wall heights normalized by $\alpha_{i}(h=1.65)=-1.1762 .(\longrightarrow) h=$ 1.65: $(\ldots \ldots) h=2.33 ;(----) h=2.70$ $(--\longrightarrow) h=3.38$ : $h--) h=3.75$. (b) Real part of pressure disturbance. (س outgoing; $(\longrightarrow)$ incoming.

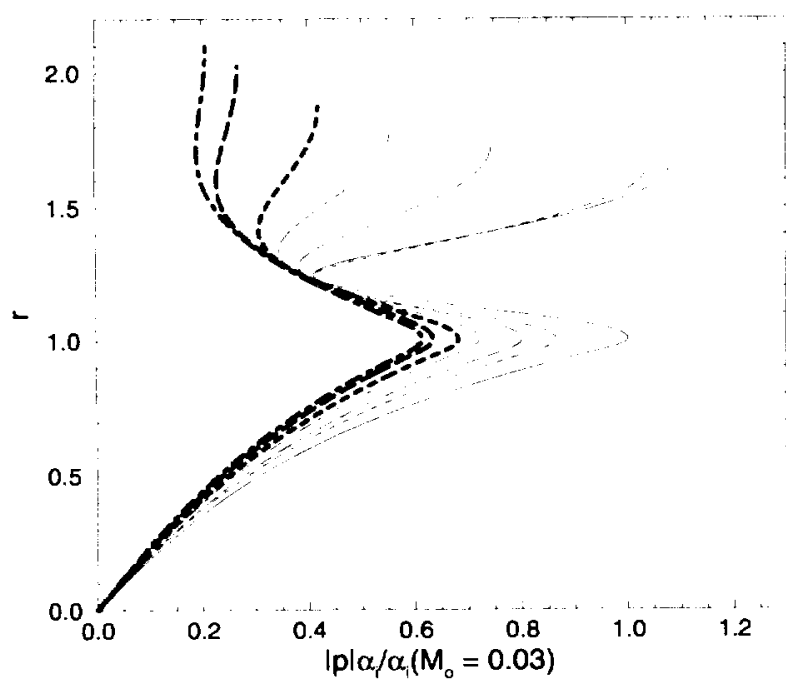

Figure 5: Radial distribution of $n=1$ wave modes at maximum growth rate with $S t=0.45$ for an $M_{j}=2.0$ jet in a hard wall cylindrical duct, $b=$ 0.05. Normalized by $\alpha_{i}\left(M_{0}=0.03\right)=-1.1762$. $\left(\stackrel{\longrightarrow}{\longrightarrow} M_{0}=0.03, h=1.65:(----) M_{0}=\right.$ $0.1, h=1.65 ;(---) M_{o}=0.2, h=1.73$; $\left.(--\longrightarrow) M_{0}=0.3 . h=1.80\right) ;(\boldsymbol{m} \boldsymbol{m} \boldsymbol{-}) M_{0}=$ $0.4 . h=1.88 ;\left(-\boldsymbol{-}, M_{0}=0.5, h=2.03 ;\right.$ $(-\infty-\infty) M_{o}=0.6, h=2.10$. spectively. The phase differences for a local minimum growth rate are $52.4^{\circ}$ for $h=2.33$ and $66.7^{\circ}$ for $h=3.38$. Thus. the pressure disturbances are more out of phase for a local maximum growth rate than for a local minimum growth rate.

As $M I_{0}$ increases, the variations in the growth rate contours in Figures 3(b) to (d) decrease and the general trend is toward lower maximum growth rates for $S t \leq 0.45$. This latter trend was observed in calculated results for both free jets with a collowing strean ${ }^{12}$ and confined cylindrical vortex sheets. The largest growth rate within the calculated region for each of the four flow conditions in Figure $3 \mathrm{oc}-$ curs at $S t=0.45$ and at a lower wall height. The radial distributions for these growth rates are shown in Figure $\bar{j}$ along with the largest growth rate results for the other three flow conditions. The relative decrease in the amplitude of the mole as $M_{\text {" increases }}$ is shown by normalizing the radial node distribution using the local growth rate. The radial distribution is multiplied by its local growth rate and then normalized by the local growth rate at $M_{0}=0.03$. The decrease in amplitude is due to the change in the direction of the disturbance wave vector as the flow increases. From equation (1), we can define a phase function for a clisturbance wave

$$
\Phi=S(\vec{r})-\omega t
$$

where

$$
S(\vec{r})=\hat{\psi}_{n}(i \lambda r)+\int_{0}^{r} \alpha_{r}(\chi) d \chi+n \theta
$$

and $\varphi_{n}(i \lambda r)$ is the real phase function for $H_{n}^{(1)}(i \lambda r)$ in the case of an outgoing disturbance wave. To follow the local wave front, $\Phi$ in equation (12) must remain constant and following Goldstejn. $1: 3$ an incromental change in $\Phi$ to lowest order is

$$
\Gamma S \cdot d \vec{r}=\omega d t
$$

From this equation, the unit normal to the wave front is defined by $\nabla S /|\Gamma S|$ with components

$$
\begin{aligned}
& {[\nabla S]_{r}=\frac{\partial S}{\partial r}=\frac{\partial \varphi_{n}}{\partial r}} \\
& {[\nabla S]_{\theta}=\frac{1}{r} \frac{\partial S}{\partial \theta}=\frac{n}{r}} \\
& {[\nabla S]_{r}=\frac{\partial S}{\partial x}=\alpha_{r}}
\end{aligned}
$$

and with direction rosines

$$
\begin{aligned}
\cos \phi_{r} & =\frac{1}{|\nabla S|} \frac{\partial \varphi_{n}}{\partial r} \\
\cos \phi_{\theta} & =\frac{1}{|\Gamma S|} \frac{n}{r} \\
\cos \phi_{y} & =\frac{\alpha_{r}}{|\Gamma S|}
\end{aligned}
$$


Using these results, we determine from the eigenfunction results in Figure 5 that the wave front direction $\phi_{x}$ changes on average from $57.00^{\circ} \pm 0.05^{\circ}$ to $42.75^{\circ} \pm 0.38^{\circ}$ as the outer flow Mach number increases from 0.03 to 0.6 . The lower angle means the wave vector points more towards the $x$-axis in the downstream direction and less of the reflecting disturbance returns to the shear layer to have any effect. As a consequence, the growth rate and the mode shape are approaching that of the free jet as $M_{o}$ increases.

Finally, the regions of supersonic or subsonic relative phase velocity are demarcated by dashed lines in the plots of Figure 3. For a phase velocity supersonic relative to the inner stream jet velocity,

$$
\left|1-c_{p h}\right|>\frac{1}{M_{j}},
$$

and for a phase velocity supersonic relative to the outer stream.

$$
\left|\bar{u}_{o}-r_{p h}\right|>\frac{\bar{u}_{O}}{M_{o}} .
$$

where $c_{p h}=\omega / \alpha$, is the local phase velocity of the instability wave. In Figures 3(a) and (b), the phase velocities are all supersonic relative to the outer flow. A small portion of the growth rate plot becomes subsonic relative to the outer flow at $M_{o}=0.3$ (lower left of part (c)). This region increases in size as $M_{o}$ increases as seen in part (d). In the small regions outlined by the thin dashed lines in parts (a) to $(c)$, the phase velocities are supersonic relative to the inner flow. Most of the growth rates lie in the region where the phase velocity is subsonic relative to the inner flow. The most important results for the wall to affect the shear layer growth rates is that the phase velocity be supersonic relative to the outer flow, especially when the wall has acoustic lining." The flow conditions chosen meet the criterion of having the phase velocity supersonic relative to the outer flow when lined walls are present.

\section{Lined Wall}

It has been previously shown that instability wave growth rates decreased as the real part of the wall impedance decreased toward one for a fixed imaginary part and wall height. ${ }^{3}$ We confirmed this behavior for the cylindrical duct case with similar operating conditions and impedance boundary conditions. In this paper, we present results for a range. of wall impedances that are found in many practical applications. The real part of the impedance is always positive with values chosen to be between 0 and 2. The imaginary part is either positive or negative with values between -2 and 2 chosen for study. These impedance values are realizable with practical sound absorbing materials.

\section{Growth Rates versus Impedance}

The initial growth rates of the $n=1$ shear layer instability wave modes were calculated for a wall height $h=2$ and a Strouhal number $S t=0.2$. With seven outer flow conditions, a large number of calculations were performed over the designated region in the impedance plane. To compare this large number of calculated results, we chose to compare growth rates affected by the presence of a wall impedance to the growth rate of a free jet shear layer. The growth rates were mapped on to the impedance plane and contours were drawn through the data correspond- (a)

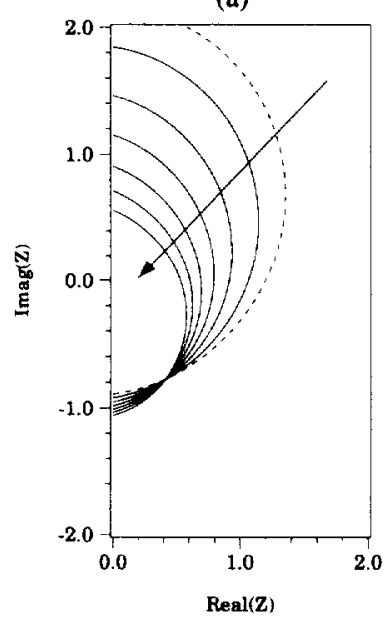

(c)

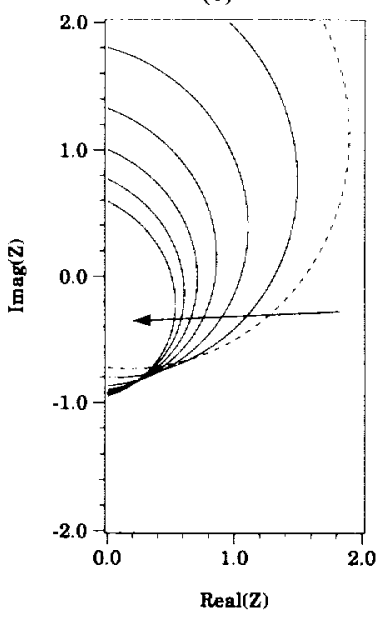

(b)

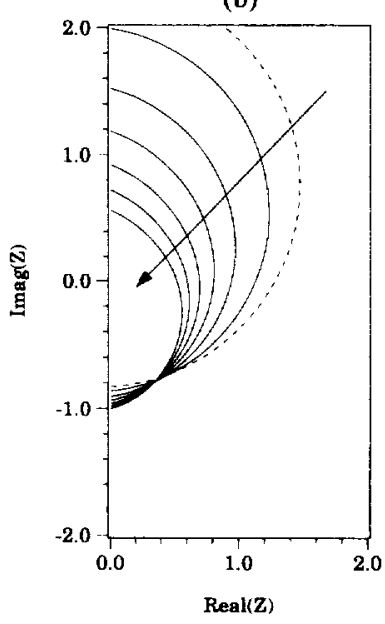

(d)

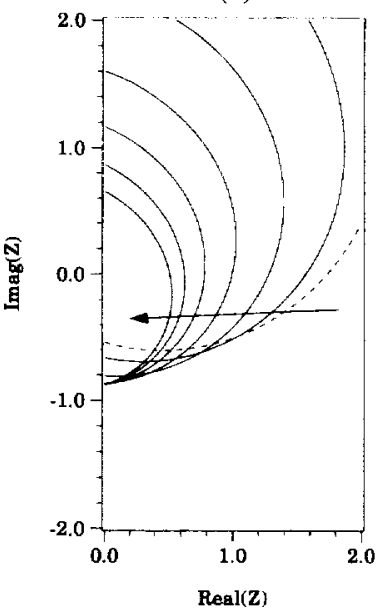

Figure 6: Local growth rates for the $n=1$ mode of an $M_{j}=2.0$ jet in a lined cylindrical duct, $h=2, S t=0.2$. Contours in the impedance plane for: (a) $b \approx 0.0$; (b) $b=0.05$; (c) $b=0.10$; (d) $b=0.15$. Contours for free jet growth rate at $M_{0}=0.03(---)$ and $M_{0}=0.1$ to 0.6 (-) following arrow. Arrow points from region where growth rates lower than free jet to region where growth rates higher than free jet. 
ing to the free jet growth rate: thus, marking the boundary between regions where the growth rate was higher than the free jet growth rate and regions where it was lower. Examples are shown in Figure 6. Each part of the figure shows seven contours corresponding to the free jet growth rates for the seven outer flow conditions at a fixed axial location determined by the jet shear layer half-width. The arrows point in the direction that the contour moves with increasing outer flow Mach number $M_{o}$ and point into the region of higher growth rates. As $M_{0}$ increases, the region of higher growth rates decreases in size and as the shear layer half-wilth increases, this area expands especially at lower $M_{0}$.

Figure 6 only shows the separate regions in the impedance plane between regions with growth rates higher or lower than that of the free jet. Figure 7 shows the range of those growth rates. For each shear layer half-width location, the figure shows the largest growth rate, the smallest growth rate, and the free jet growth rate within that calculated region of the impedance plane. As $b$ increases, the growth rates generally decrease and as $M_{o}$ increases. the growth rates decrease and the range of increased growth rates, the difference between the largest growth rate and the free jet growth rate, also docreases. The largest growth rates shown in Figure 7 lie along the inaginary axis of the impedance planes in Figure 6 near the center of a circle that approximates the shape of the free jet growth rate contours.

Figure 8 shows how the free jet growth rate contours change in the impedance plane when the duct wall height is changed with $b=0.05 . S t=0.2$, and $n=1$. The duct wall height $h$ starts at $1.6 \mathrm{and}$ progresses to $2.0,2.5$, and 3.0 . At $h=1.6$, the contours change location a small amount. As $h$ increases, the contours unfold creating larger regions of higher growth rates. The contours at lower $h$ are

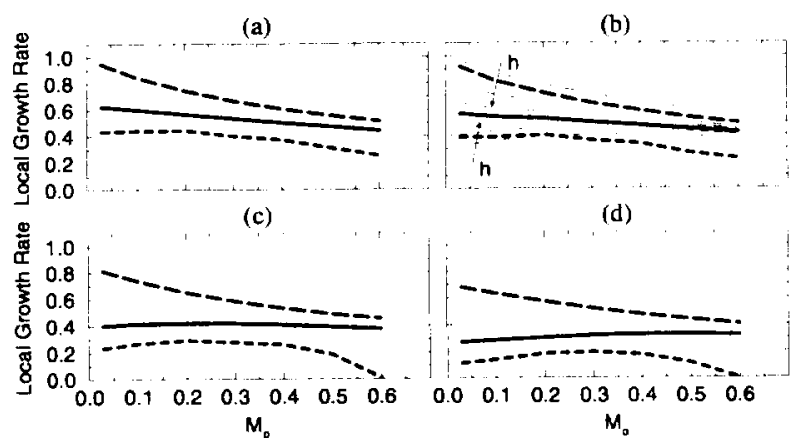

Figure 7 : Range of local growth rates for conditions in Figure 6. (

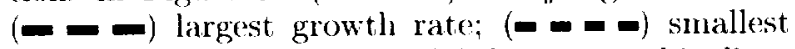
growth rate. (a) $b \approx 0.0$; (b) $b=0.05$, thin lines correspond to conditions in Figure 8; (c) $b=0.01$; (d) $b=0.15$. circular in shape, wrapping around the point of the largest growth rate. The contours unfold as $h$ increases until they start to wrap around the point of the smallest growth rate. This also may be viewed as a movement of the largest growth rate point toward larger positive inaginary impedance values, outside the impedance range shown. as $h$ increases and a movement of the smallest growth rate point into the region from more negative imaginary impedance valnes. There may be more local maximum and minimum growth rate points that lie outside this region of the impedance plane. Even though the region of increased growth rates get larger as $h$ increases, the (a)

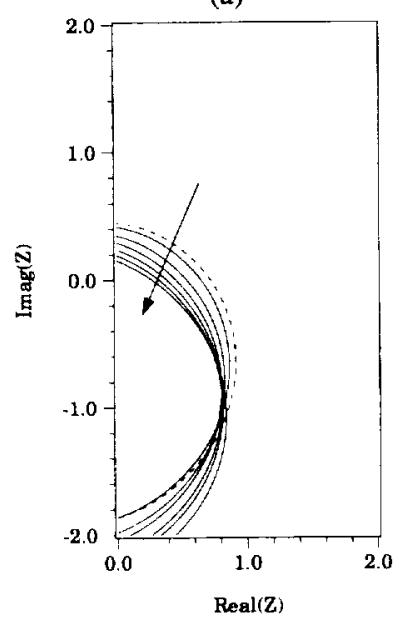

(c)

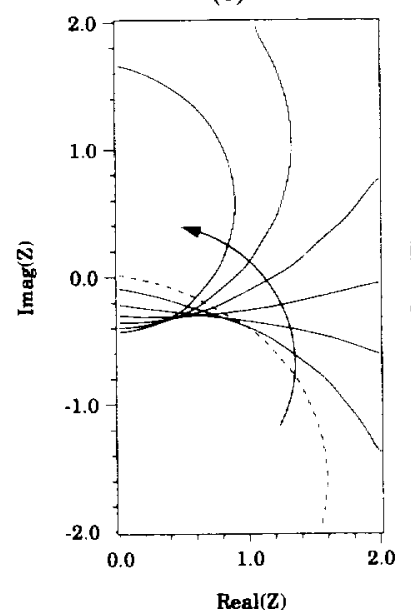

(b)

(d)

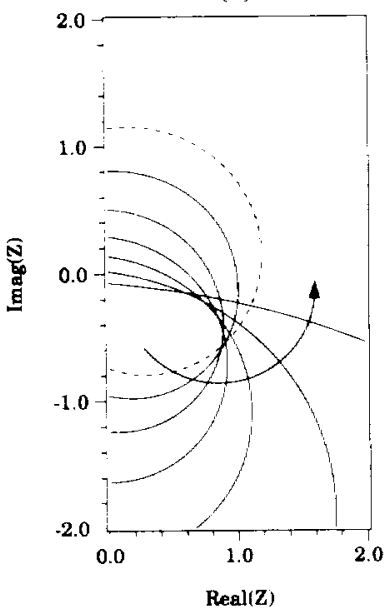

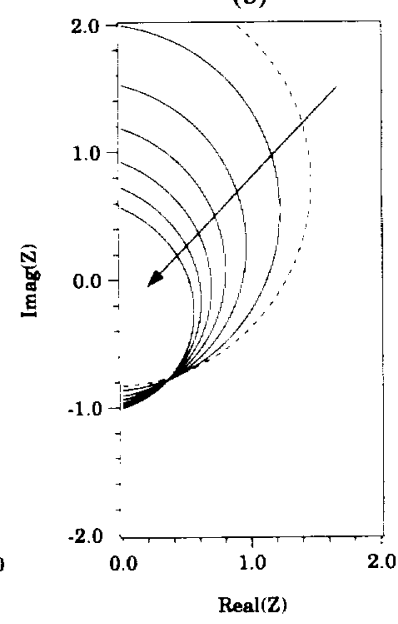

Figure 8: Local growth rates for the $n=1$ mode of an $M_{j}=2.0$ jet in a lined rylindrical duct, $b=0.05$. St $=0.2$. Contours in the impedance plane for: (a) $h=1.6$; (b) $h=2.0$; (c) $h=2.5$; (d) $h=3.0$. Contours for free jet growth rate at $M_{0}=0.03(---)$ and $M_{0}=0.1$ to 0.6 $(\longrightarrow$ following arrow. Arrow points from region where growth rates lower than free jet to region where growth rates higher than free jet. 
range of those growth rates decrease as shown in Figure $7(b)$. The increasing outer flow decreases the range even more until for the $M_{o}=0.6$ growth rates in the impedance plane of Figure $8(\mathrm{~d})$, there is only a $10 \%$ change from smallest to largest growth rates at $h=3.0$.

The results for the $n=2$ helical mode at $S t=0.2$ follow the same trends as those seen for the $n=1$ mode above though with generally higher growth rates. An example of the calculated growth rate results in the impedance plane is shown in Figure 9 in terms again of the free jet growth rate contours. These plots for $b=0.05$ and $h=2$ should be compared to the $n=1$ results shown in Figures $6(\mathrm{~b})$ and $7(b)$.

Calculations were also performed at a Strouhal number of 0.3 , a duct wall height of 2 , and a shear layer half-width of 0.05 . The free jet growth rate contours are shown in Figures 10(a) and (b) for the $n=1$ and $n=2$ modes, respectively. The corresponding growth rate ranges as a function of $M_{0}$ are shown in parts (c) and (d). Note the similarity between Figures 10(a) and $8(c)$. The duct wall heights and Strouhal numbers for these two cases are such that $S t(h-1)$ is a constant. This indicates that an important length scale near the nozale may be the jet radius to duct wall distance. However, the growth rates may be higher and the range of growth rates is certainly larger for the $S t=0.3$ case than for the $S t=0.2$ case, compare Figure $10(c)$ to Figure $7(b)$ with $h=2 . \bar{j}$.

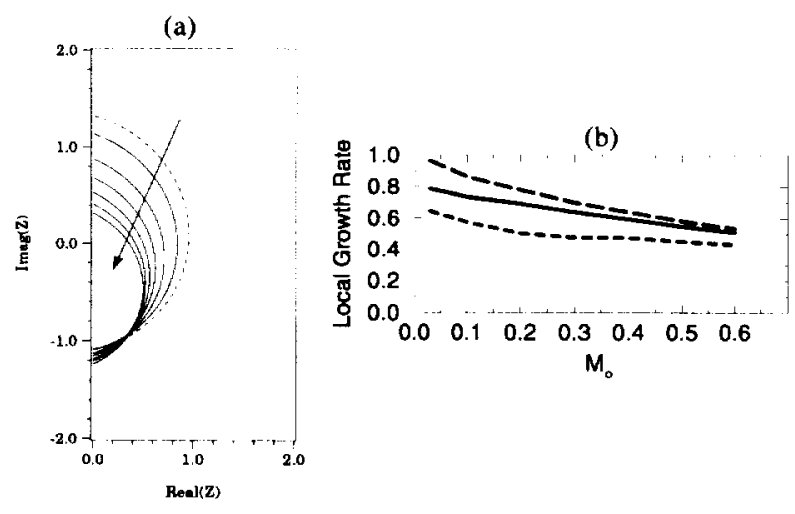

Figure 9: Local growth rates for the $n=2$ mode of an $M_{j}=2.0$ jet in a lined cylindrical duct, $h=2, S t=0.2, b=0.05$. (a) Contours in the impedance plane for free jet growth rate at $M_{o}=$ $0.03(---)$ and $M_{o}=0.1$ to 0.6 ( lowing arrow. Arrow points from region where growth rates lower than free jet to region where growth rates higher than free jet. (b) Range of local growth rates. (

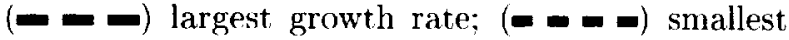
growth rate.
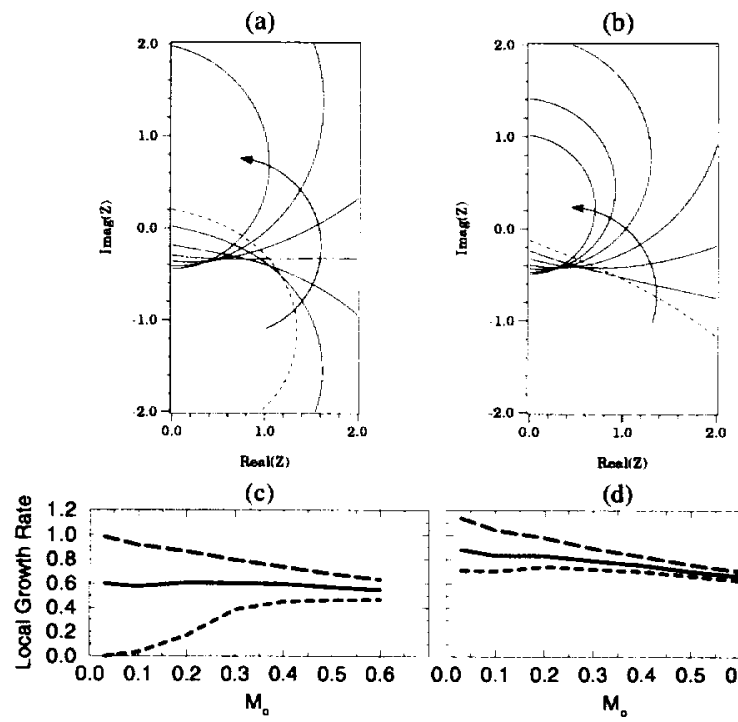

(d)

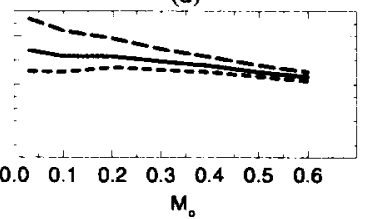

Figure 10: Local growth rates of an $M_{j}=2.0$ jet in a lined cylindrical duct, $h=2$. St $=0.3$, $b=0.05$. Contours in the impedance plane: (a) $n=1$; (b) $n=2$. Contours for free jet growth rate at $M_{o}=0.03(----)$ and $M_{o}=0.1$ to 0.6 $(\longrightarrow)$ following arrow. Arrow points from region where growth rates lower than free jet to region where growth rates higher than free jet. Range of local growth rates: (c) $n=1 ;$ (d) $n=2$. (- free jet growth rate; ( $-\boldsymbol{D}$ ) largest growth rate; $(\mathbf{-}-\mathbf{m})$ smallest growth rate.

\section{Growth Rates versus $h$ and $\mathbf{S t}$}

The previous results fixed the geometry and flow parameters and explored shear laver growth rates as a function of the wall impedance. Next, we select a wall impedance and calculate the growth rates as a function of wall height and Strouhal number. Using the results for $M_{0}=0.3$ as an example. Figure 11 shows growth rate contours for a set of impedance values. In the horizontal direction from left to right, the real part is $0.4,1.0$, and 1.5 and in the vertical direction from bottom to top, the imaginary part is $-0.8,0.0$, and 0.8 . At $h=4.5$, the growth rate contours on all the plots in the figure are nearly the same and mostly vertical indicating that the growth rates are about the same as the free jet growth rates since the effects of reflections are negligible. As $h$ decreases, the effects of the lined wall on growth rates first appear at higher Strouhal numbers and then progress to lower Strouhal numbers. The effects are larger, meaning higher maximum growth rates and larger variations in growth rates as the wall height changes, when the imaginary part of the impedance is positive and for smaller real parts of the impedance.

When the outer flow changes, the previous results 
(a)

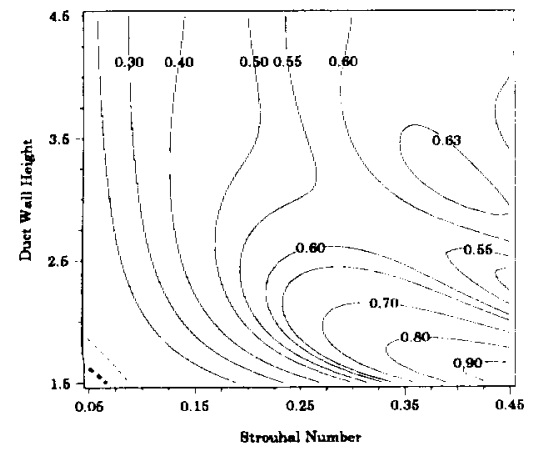

(d)

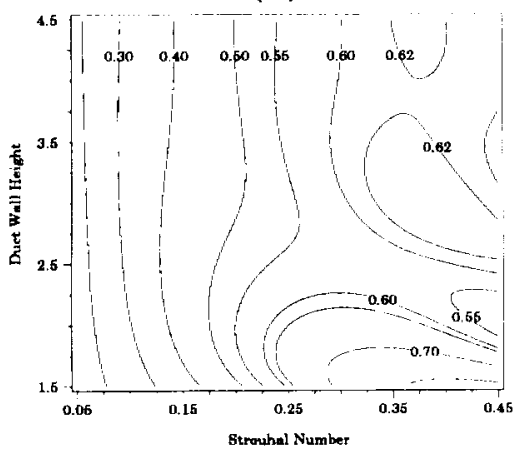

(g)

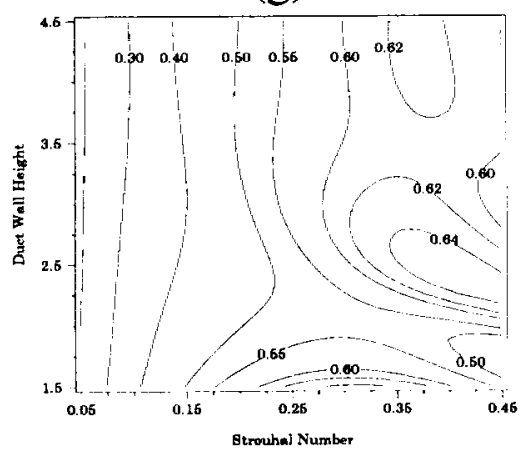

(b)

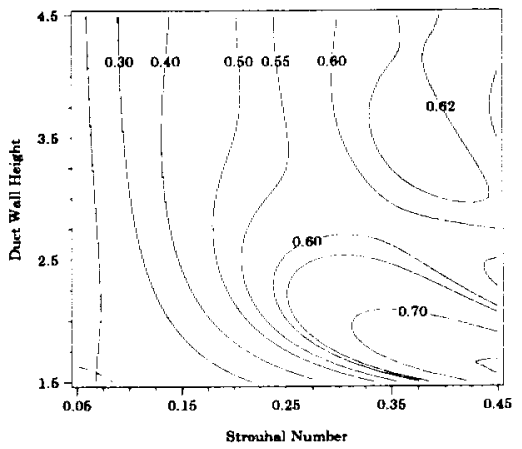

(e)

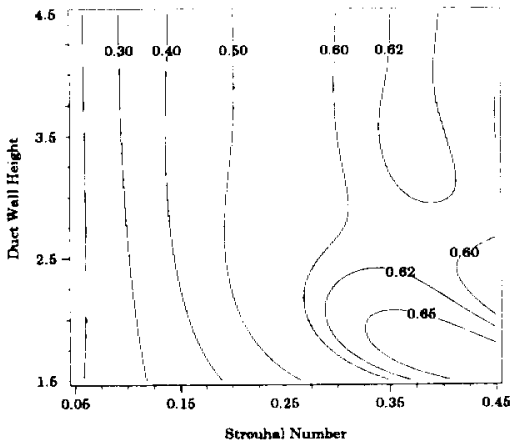

(h)

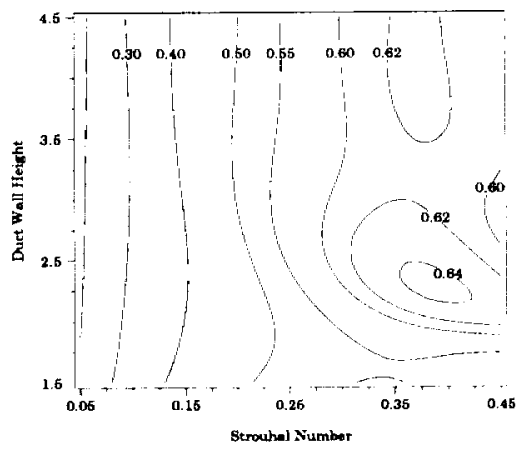

(c)

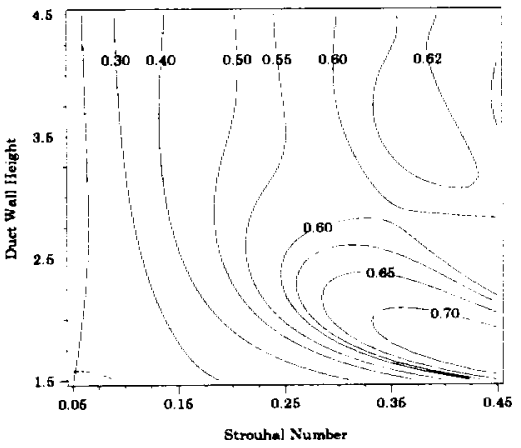

(f)

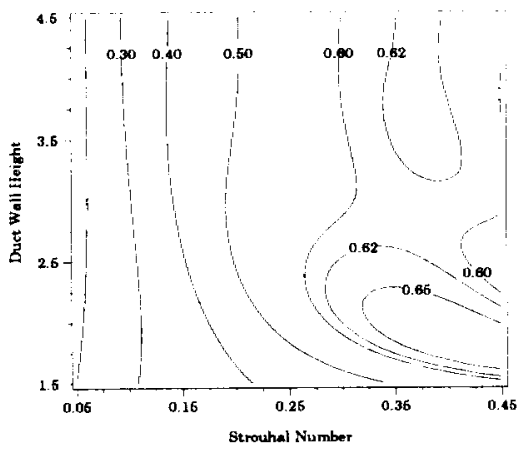

(i)

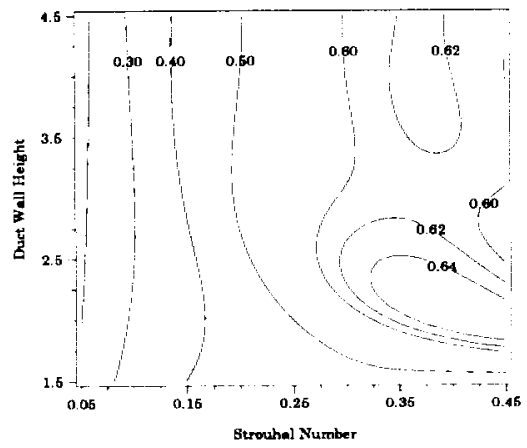

Figure 11: Local growth rate contours as a function of $h$ and $S t$ for the $n=1$ mode of an $M_{j}=2.0$ jet in a lined cylindrical duct, $M_{o}=0.3, b=0.05$. (a) $Z=0.4+i 0.8$; (b) $Z=1.0+i 0.8$; (c) $Z=1.5+i 0.8$; (d) $Z=0.4$; (e) $Z=1.0$; (f) $Z=1.5$; (g) $Z=0.4-i 0.8$ : (h) $Z=1.0-i 0.8$ : (i) $Z=1.5-i 0.8$.

have shown that the growth rate changes for each wall impedance. If we now fix the wall impedance at the extremes of effect in Figure 11, that is $Z=$ $0.4+i 0.8$ where there were large variations in growth rates and $Z=1.5-i 0.8$ where there were small variations in growth rates, the effects of external flow on growth rates can be shown as a function of wall height and Strouhal number. These results are shown in Figures 12 and 13. The four flow conditions shown in each figure are $M_{o}=0.03,0.1,0.3$, and 0.6. The $M_{o}=0.3$ results are the same as shown in Figure 11. Thus, we see with lower $M_{\text {, }}$ that there are much larger variations in growth rate and less variations as $M_{0}$ increases above 0.3 . The general trends in growth rates discussed for $M_{0}=0.3 \mathrm{in}$ Figure 11 also occur at the higher and lower outer flow Mach numbers. Figures 12 and 13 can also be compared to the hard wall results shown in Figure 3. Figure 12 shows generally increased growth rates as the wall height decreases when compared to the hard wall cases. We find the opposite or generally decreased growth rates when Figure 13 is compared to Figure 3.

\section{CONCLUDING REMARKS}

For a hard wall confining duct. this analysis con- 


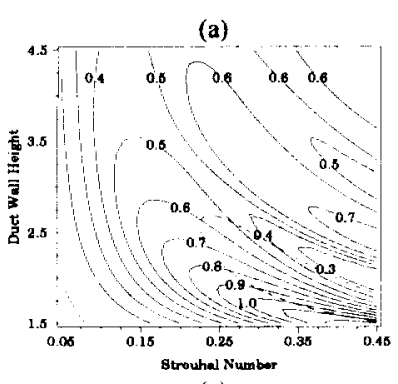

(c)
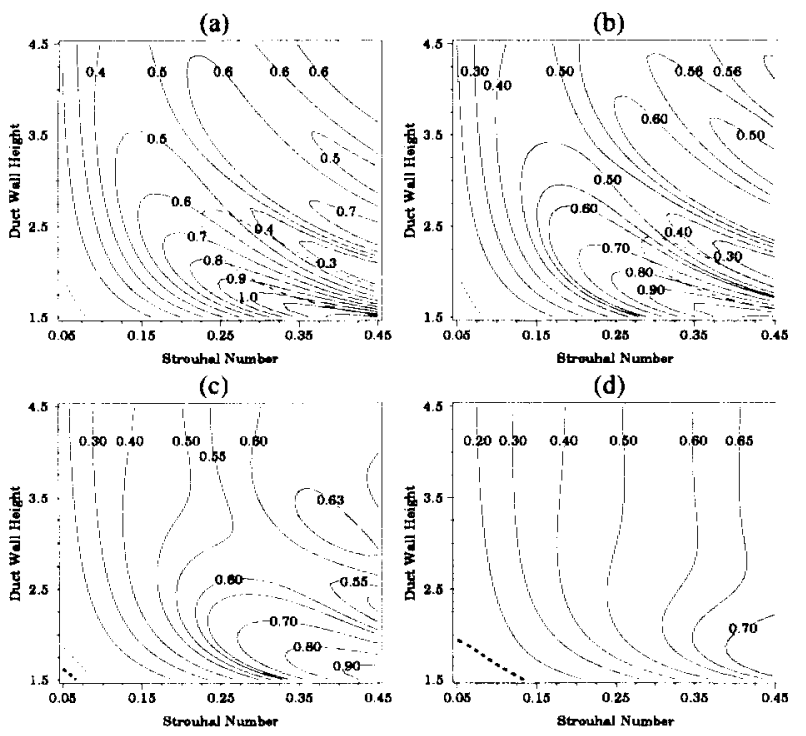

(d)

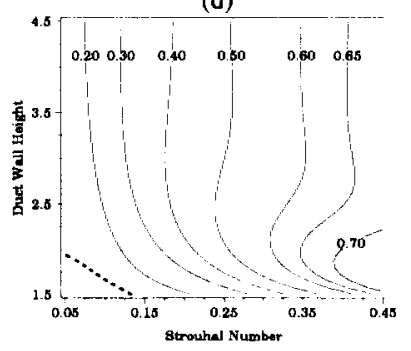

Figure 12: Local growth rate contours as a function of $h$ and $S t$ for the $n=1$ mode of an $M_{j}=2.0$ jet in a lined cylindrical duct with $Z=0.4+i 0.8$, $b=0.05$. Outer flow: $M_{o}=$ (a) 0.03 ; (b) 0.1 ; (c) 0.3; (d) 0.6. Phase velocities supersonic relative to outer flow and subsonic relative to inner flow except inside thick dashed line (subsonic re. onter flow) and inside thin dashed line (supersonic re inner flow).

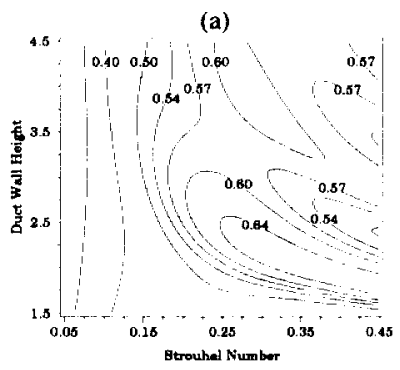

(c)
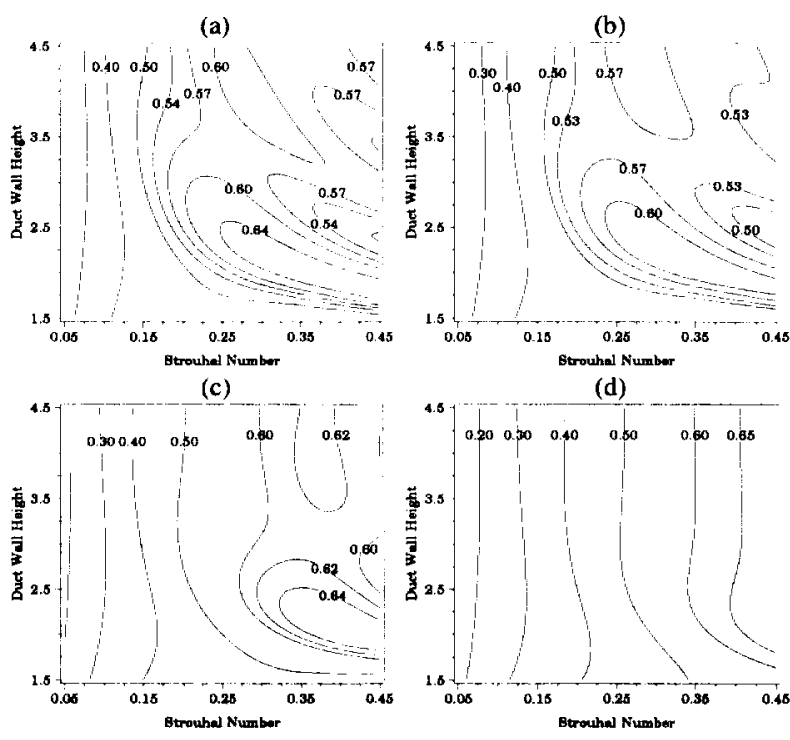

(d)

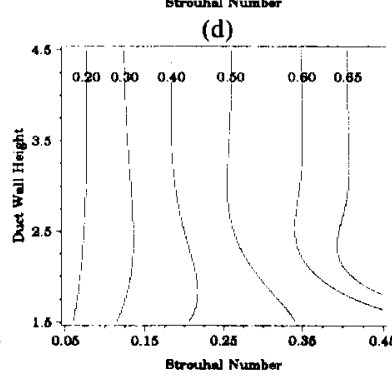

Figure 13: Local growth rate contours as a function of $h$ and $S t$ for the $n=1$ mode of an $M_{j}=2.0$ jet in a lined cylindrical duct with $Z=1.5-i 0.8$, $b=0.05$. Outer flow: $M_{o}=$ (a) 0.03 ; (b) 0.1 ; (c) 0.3 ; (d) 0.6 . Phase velocities supersonic relative to outer flow and subsonic relative to inner flow. firmed that the growth rates of Kelvin-Helmholtz type instability waves were affected by wall height when the waves had phase velocities supersonic relative to the outer flow. The results presented here agreed with previous calculated results for the cylindrical vortex sheet case in a hard wall duct where the growth rates generally decrease with an increase in the outer flow. However, as the wall height and Strouhal number change, the growth rate oscillated between local minimums and local maximmms. The range between minimum and maximum decreased as the outer flow increased. The phase relationship between the outgoing disturbance and the reflected incoming disturbance was a factor in determining if the growth rate was a local minimum or a local maximum. The phase difference was approximately $-150^{\circ}$ just outside the shear layer for a local maximum growth rate and approximately $60^{\circ}$ for a local minimum growth rate. As the outer flow increased, it was shown that the pressure disturbance wave vector shifted toward the downstream direction. so that for a fixed wall height less reflected disturbance affected the growth rate. Further analysis using the wave vector would reveal direction and level of disturbance energy.

The calculated results for the initial growth rates for a jet shear layer in a lined duct were presented for changes in wall impedance, wall height, outer flow, frequency or Stroulal number, and mode number. With all conditions fixed except for variations in the wall impedance, the growth rate could be made to increase above or decrease below the growth rate for the free jet. Within the region of the impedance plane used in the calculations, a maximum and a minimum growth rate existed for the fixed condition. This range of growth rates was decreased when the outer flow increased at constant shear layer width. At fixed flow conditions, an increase in shear layer width lowered the growth rates but the range of growth rates changed little.

For a fixed impedance, flow condition, and mode number, growth rate variations were calculated as the wall height and Strouhal number were varied. In general for all the flow conditions studied here, these growth rate variations decreased when the inaginary part of the impedance was less than zero and increased when the imaginary part of the impedance was greater than zcro. With the varied combinations of wall height, Strouhal number, flow conditions, and wall impedance, a particular growth rate can be higher or lower than the growth rate for a free jet or for a jet in a hard wall duct. If it is desirable to lower growth rates in general, then those modes and frequencies that may tend to grow the most should be reduced. Wall impedance values with negative imaginary values can be chosen to reduce the initial growth rates of these modes. 


\section{REFERENCES}

1. D. S. Shin and J. H. Ferziger. Linear stability of the confined compressible reacting mixing layer. AIAA I.. 31:571 57\%. 1993.

2. K. K. Aluja, K. C. Massey. A. J. Fleming. C. K. W. Tam, and R. R. Jones III. Acoustic interactions between an altitude test facility and jet engine plumes theory and experiments. Technical Report TR-91-20, AEDC. 1992.

3. F. Q. Hu. The acoustir and instability waves of jets contined inside an acoustically lined rectangular duct. J. Sound Vib., 183:841 856, 1995.

4. C. K. W. Tam and F. Q. Hu. The instability and acoustic wave modes of supersonic mixing layers inside a rectangular channol. J. Fluid Mech., 203:51 76, 1989.

5. M. Zhuang, P. E. Dimotakis, and T. Kulbota. The effect of walls on a spatially growing supersonic shear layer. Phys. Fluirls A, 2:599-604. 1990 .

6. P. J. Morris and M. G. Giridharan. The effect of walls on instability waves in supersonic shear layers. Phys. Fluids A, 3:356 358, 1991.

7. K. Viswanathan. Turbulent Mixing in Supersonic: Jets. PhD thesis, Penn State University, 1991.

8. C. C. Chang and C. Y. Kuo. Instability of a supersonic vortex sheet inside a circular duct. Phys. Fluids A, 5:2217 2228, 1993.

9. K. Viswanathan, P. J. Morris, and G. Chen. Linear instability waves in supersonic jets confined in circular and non-circular ducts. $J$. Sound Vib. 171:231 253. 1994.

10. C. K. W. Tam and D. E. Burton. Sound generated by instability waves of supersonic flows. part 2. axisymmetric jets. J. Fluid Mech. 138:273-295, 1984.

11. M. D. Dahl. The Aeroucoustics of Supersonic Coaxial Jets. PhD thesis, Pem State University, 1994.

12. A. Michalke and G. Hermann. On the inviscid instability of a circular jet with external flow. J. Fluid Mech., 114:343-359, 1982.

13. M. E. Goldstein. Aeroucoustirs. McGraw-Hill, New York, 1976. 
Public reporting burden for this collection of information is estimated to average 1 hour per response, including the time for reviewing instructions, searching existing data sources, gathering and maintaining the data needed, and completing and reviewing the collection of information. Send comments regarding this burden estimate or any other aspect of this collection of information, including suggestions for reducing this burden, to Washington Headquarters Services, Directorate for Information Operations and Reports, 1215 Jefferson Davis Highway, Suite 1204, Arlington, VA 22202-4302, and to the Otfice of Management and Budget, Papenwork Reduction Project (0704-0188), Washington, DC 20503.

\begin{tabular}{|l|c|c|c|}
\hline 1. AGENCY USE ONLY (Leave blank) & $\begin{array}{c}\text { 2. REPORT DATE } \\
\text { May } 1997\end{array}$ & $\begin{array}{c}\text { 3. REPORT TYPE AND DATES COVERED } \\
\text { Technical Memorandum }\end{array}$
\end{tabular}

4. TITLE AND SUBTITLE

5. FUNDING NUMBERS

Effects of Acoustically Lined Cylindrical Ducts on Instability Waves in Confined Supersonic Jets

6. AUTHOR(S)

WU-505-62-52

Milo D. Dahl

7. PERforming ORganization NAME(S) AND ADDRESS(ES)

8. PERForming ORGANIzATION REPORT NUMBER

National Aeronautics and Space Administration

Lewis Research Center

Cleveland, Ohio 44135-3191

E-10709

9. SPONSORINGMONITORING AGENCY NAME(S) AND ADDRESS(ES)

10. SPONSORINGMONITORING AGENCY REPORT NUMBER

National Aeronautics and Space Administration Washington, DC 20546-0001

NASA TM-107441

AIAA-97-1600

\section{SUPPLEMENTARY NOTES}

Prepared for the Third Aeroacoustics Conference sponsored by the American Institute of Aeronautics and Astronautics and the Confederation of European Aerospace Societies, Atlanta, Georgia, May 12-14, 1997. Responsible person, Milo D. Dahl, organization code 2660, (216) 433-3578.

\begin{tabular}{|l|l|l|l|l|} 
12a. DISTRIBUTIONAVAILABILTY STATEMENT & 12b. DISTRIBUTION CODE
\end{tabular}

Unclassified - Unlimited

Subject Category 34

This publication is available from the NASA Center for AeroSpace Information, (301) 621-0390.

13. ABSTRACT (Maximum 200 words)

The pressure disturbances generated by an instability wave in the shear layer of a supersonic jet are studied for an axisymmetric jet inside a lined cylindrical duct. For the supersonic jet, locally linear stability analysis with duct wall boundary conditions is used to calculate the eigenvalues and the eigenfunctions. These values are used to determine the growth rates and phase velocities of the instability waves and the radial pressure disturbance patterns. The study is confined to the dominant Kelvin-Helmholtz instability mode and to the region just downstream of the nozzle exit where the shear layer is growing but is still small in size compared to the radius of the duct. Numerical results are used to study the effects of changes in the outer flow, growth in the shear layer thickness, wall distance, wall impedance, and frequency. Results indicate that the effects of the duct wall on shear layer growth rates diminish as the outer flow increases. Also, wall reflections cause variations in growth rates depending on wall height and Strouhal number. These variations are due to the phase relationship between the outgoing and the reflected incoming pressure disturbances at the shear layer. The growth rate variations can be reduced and the maximum growth rate minimized by keeping the imaginary part of the impedance negative.

\section{SUBJECT TERMS}

Supersonic jets; Acoustic treatment; Instability waves; Ejectors; Confined jets

\begin{tabular}{|c|c|}
\hline $\begin{array}{c}\text { 17. SECURITY CLASSIFICATION } \\
\text { OF REPORT } \\
\text { Unclassified }\end{array}$ & $\begin{array}{c}\text { 18. SECURITY CLASSIFICATION } \\
\text { OF THIS PAGE } \\
\text { Unclassified }\end{array}$ \\
\hline
\end{tabular}

19. SECURITY CLASSIFICATION OF ABSTRACT Unclassified
15. NUMBER OF PAGES

13

16. PRICE CODE

A03

20. LIMITATION OF ABSTRACT 